\title{
Discussion on Piezoelectric Power Generation System
}

\author{
Xiaoming Sun \\ Chongqing Water Resources and Electric Engineering College, Chongqing, Yongchuan 402160, China
}

\begin{abstract}
Coal, petroleum and natural gas will still be the basis of economic development for a long time. However, with a rapider consumption speed, these fossil fuels will be exhausted in the near future. In addition, the usage of these fossil fuels can also cause environmental pollution and greenhouse effect. To deal with energy security and environmental crisis, it is wise to work towards three directions: energy saving and emission reduction, energy recovery, exploration of new renewable energy. Currently, the electricity generation technology using piezoelectric material to recover the compressional or vibrational energy begins to draw attention. However, most of the researches are devoted to designing small self-powered devices. This paper presents an overview of the feasibility of piezoelectric power generation system for electric power system, in which the fundamentals of piezoelectric power generation and the feasible structure of the system are discussed.
\end{abstract}

Keywords—piezoelectric power generation; renewable energy; electricity generation

\section{INTRODUCTION}

Coal, petroleum and natural gas will still be the basis of economic development for a long time. However, with a rapider consumption speed, these fossil fuels will be exhausted in near future. In addition, the usage of these fossil fuels can also cause environmental pollution and greenhouse effect [1] To deal with energy security and environmental crisis, it is wise to work towards three directions: energy saving and emission reduction, energy recovery, exploration of new renewable energy.

Although wind power generation and solar power generation are the eminent achievements of the above work, their development is restricted for the following reasons. First, for China, except western areas, the resources of wind power and solar power in most of the mainland are not abundant, the exploitation and utilization of which are not economic. Second, the electrical distance from the western wind farms and solar farms to the central and eastern load centers is very large, therefore, with the limitations of transient stability or dynamic stability, the power of the AC transmission lines can not reach the natural transmission capacity, which may produce "abandoned wind" and "abandoned sunlight"; if the energy is transmitted by DC transmission lines instead, considering the high cost of DC transmission lines, the method can not be used widely. Third, when penetration rate increases, to balance the power fluctuation caused by the intermittency of wind and sunlight, the peak load regulation capacity distributed to thermal power units will increase as well, and this increases the extra cost of coal and thus reduces the low carbon benefit of wind power and solar power [2]. Fourth, large-scale wind farms and solar farms tend to occupy a very large area, and even urban-type equipment need also enough spaces to install; moreover, wind power generator may induce noise pollution [3] and solar power generation equipment may induce light pollution; therefore, it is not very appropriate to install wind power generator and solar power generation equipment in cities with high concentration of population and construction.

Recently, the electricity generation technology using piezoelectric material to recover the compressional or vibrational energy begins to draw attention. The principle is based on the positive piezoelectric effect of piezoelectric material: when the piezoelectric material deforms under the external force (pressure or stress), the inside polarization phenomenon occurs, and the charges of different polarity accumulate on two opposite surfaces; when the external force disappears, the charges disappear accordingly. If intermittent external force is continuously exerted on piezo-electric material and the charges appearing on the surfaces are simultaneously collected by charge collector and stored in energy storage equipment, the transition from mechanical energy to electric energy is realized. In this process, there are no emission, no electromagnetic conversion, no heat and no big mechanical vibration; therefore, piezoelectric power generation is a new type of green power generation without pollution, electromagnetic interference (EMI), thermal radiation and noise.

In addition to the advantages above, piezoelectric power generation equipment has many other advantages-simple structure, light weight, low cost, large energy density, long service life and easy integration, which overcome the shortcomings of wind power generation and solar power generation. Piezoelectric power generation is more suitable for using in cities, and because it is almost unaffected by climate and weather, it can be applied throughout the nation without any regional limitations. Piezoelectric power generation equipment can be integrated with buildings or facilities, not affecting landscape and occupying extra area and space. For example, if integrated with floor tile or pavement [4], piezoelectric material can generate electricity when pedestrians or vehicles pass by; if integrated with high-rise building, billboard or traffic light, piezoelectric material can recover the energy of wind or noise [5] to generate electricity. Provided that these distributed piezoelectric power generation equipment can be integrated into piezoelectric power generation system and then connected to electric power grid, in cities the closely packed buildings, crowded streets and countless facilities can all be turned into huge, intangible electric power plants. Not only can these electric power plants recover the biomass energy of men, but also recover the kinetic energies of vehicles and wind. As a result, this not only generates electricity, but also damps vibration, reduces nose and thus improve city environment indirectly. Piezoelectric power generation system 
is actually distributed throughout the city, going deep into the load center and shortening the electrical distance, therefore, there almost exist no transient stability and dynamic stability problems, avoiding the problem of competing with important power plants for transmission channels.

In short, piezoelectric power generation has a wide range of application prospects in electricity generation area, and there are considerable social and economic benefits in the industrial chain from electrical equipment manufacture to electricity generation. The purpose of this paper is to present a discussion of the feasibility of piezoelectric power generation system for electricity generation, in which the fundamentals of piezoelectric power generation and the feasible system structure are discussed, which serves as a basis of the subsequent researches.

\section{Choice of Piezoelectric Material}

Piezoelectric power generation system is required to have high power generation capacity, high reliability and high stability, because only high power generation capacity can bring economical efficiency, only high reliability can bring low maintenance cost and only high stability can bring easy control and wide application. All these requirements firstly depend on the piezoelectric material adopted. At present, piezoelectric materials include piezoelectric ceramic, glass ceramic, piezoelectric crystal, piezoelectric polymer, piezoelectric composite, ferroelectric piezoelectric crystal, non-ferroelectric piezoelectric crystal, relaxation electric crystal and inorganic piezoelectric ferroelectric thin films, etc. Their power generation capacity is assessed by piezoelectric strain constant, reliability is assessed by flexibility or mechanical strength, and stability is assessed by the stabilities of performance parameters (e.g. relative dielectric constant) including temperature stability, humidity stability, chemical stability and time stability. References [6], [7] and other design manuals provide the design parameters of various types of piezoelectric materials, and they serve as important evidences at the early stage of the design. It is required that the piezoelectric material to be used is of high piezoelectric strain constant, fine flexibility or mechanical strength and stable performance parameters, and $\mathrm{PbZrO} 3-\mathrm{PbTiO} 3-5$ (PZT-5) piezoelectric ceramic is a good example.

\section{Assembling Of Piezoelectric Power Generation EQUIPMENT}

In addition to piezoelectric material, power generation capacity, reliability and stability also depend on the assembling of piezoelectric power generation equipment. The power generation capacity of piezoelectric power generation is assessed by electromechanical coupling coefficient, which indirectly reflects the transition efficiency from mechanical energy to electric energy. Piezoelectric power generation equipment is required to have high electromechanical coupling coefficient; thus, some efforts have to be made to improve the vibration mode, support mode, motivation mode and interconnection mode of piezoelectric vibrator.

As to vibration mode, 31-mode (the stretching vibration along length direction) is easy to assemble and has low system natural frequency and relatively high vibration [8], and 33mode (the stretching vibration along thickness direction) has high electromechanical coupling coefficient but is not easy to produce strain. From the principles of physics, the mathematical models of 31-mode and 33-mode can be constructed [9], [10], and then the mathematical formula of electromechanical coupling coefficient, quality factor, input energy, output energy and efficiency can be derived. Hereinto, high quality factor means low extra heat loss and more generation of electricity.

As to support mode, cantilever support and simple support are convenient to install and easy to realize. The deformation of rectangle-section cantilever support concentrates on the root, which greatly shortens the effective use length of piezoelectric material. Therefore, to promote power generation capacity, the section of rectangle-section cantilever support can be replaced by other sections of different shapes, e.g. triangle and trapezoid [11]. The deformation of triangle-section or trapezoid-section cantilever support distributes more widely and more uniformly, which can generate more electricity energy using the piezoelectric material of the same volume. Reference [12] presents a new structure of simple support for circular piezoelectric vibrator, making the deformation of piezoelectric material more uniformly than that of traditional staked configuration and thus expanding the electricity generation area.

As to motivation mode, inertial free vibration mode can recover weak environmental vibration energy (e.g. wind power and noise energy), and forced vibration mode can recover great mechanical pressure energy (e.g. vehicle crushing energy). However, only with the aid of percussion hammer (e.g. metal ball) can shock free vibration mode generate electricity; therefore, this mode may not only reduce the service life of piezoelectric power generation equipment, but also produce noise, and can only be used in situations where transient voltage or current is needed. Therefore, only inertial free vibration mode and forced vibration mode can be adopted by piezoelectric power generation system. After motivation mode is determined, the next task is to determine the resonant frequency under this motivation mode, because only when piezoelectric vibrator resonates with external force can electricity generation be maximized. To maintain the maximum electricity generation, resonant frequency should be able to be adjusted online so as to adapt to operation mode changes and various disturbances. According to whether the extra energy is needed or not, the adjusting method of resonant frequency is divided into active self-adjusting method and passive selfadjusting method. Active self-adjusting method that needs extra energy may increase electricity generation by about $30 \%$ [13], [14], however, the additional adjustment circuit may consume much more electricity energy than the increased electricity energy generated by resonant frequency adjustment. Passive self-adjusting method that does not need extra energy is no better than active self-adjusting method in increasing electricity generation [15]; on the contrary, volume is increased and structure is complicated. Therefore, the self-adjusting method of resonant frequency requires in-depth study.

As to interconnection mode, by series mode, parallel mode or series-parallel mixed mode, several piezoelectric vibrators can be interconnected to form multilayer piezoelectric vibrator 
so as to enable small piezoelectric material to have relatively high power generation capacity under weak motivation and in wide frequency band. $n$ pieces of paralleled piezoelectric vibrators increase the output current by $n$ times, and $n$ pieces of serialized piezoelectric vibrators increase the output voltage by $n$ times. Paralleled piezoelectric vibratos have great output current and great equivalent capacitors, and are applicable to small load impedance situation; serialized piezoelectric vibratos have high output voltage and small equivalent capacitors, and are applicable to great load impedance situation. Therefore, through the optimization of the series-parallel mixed mode of piezoelectric vibrators, the voltage and current output characteristics of piezoelectric power generation equipment can be optimized, which is beneficial for electric power grid connection. From analytical calculations, reference [16] demonstrates that the multilayer piezoelectric vibrator consisting of 145 piece series-parallel piezoelectric vibrators (height $1.8 \mathrm{~cm}$, section area $1 \mathrm{~cm}^{2}$ ) has $1 \sim 10 \mu \mathrm{F}$ equivalent capacitance and about $30 \mathrm{~V}$ open circuit voltage, and its matched load resistance is several kilohms. Reference [17], by changing the direction of the traditional interconnection mode, promotes the electricity energy output quantity and the efficiency by $1.5 \sim 1.8$ times.

To sum up, the assembling items of piezoelectric power generation equipment and the functions of each item are summarized in Figure 1.

\section{Charge Collector AND Energy Storage EQUIPMENT}

Considering that the charges generated by piezoelectric vibrator during each vibration period are finite, the design of high efficient charge collector and energy storage equipment is also very important for promoting efficiency and reducing energy loss. In addition, because the output of piezoelectric vibrator is an alternating current with small current and high voltage, in order to ensure the high efficient transmission of electricity energy from piezoelectric vibrator to energy storage equipment, charge collector should not only decrease the output voltage and increase the output current, but also match the equivalent load of energy storage equipment. AC-DC converter (rectifier) or AC-DC-DC converter (rectifier + chopper) can be adopted to realize the charge collector having the two functions above. The synchronous charge collecting technology [18], [19], which synchronizes the charge collecting period with the vibration period of piezoelectric vibrator, can reduce the damping brought by inverse piezoelectric effect and promote the efficiency of piezoelectric power generation equipment by $4 \sim 9$ times. It is verified by experiment that the output power of AC-DC-DC converter is almost 3 times greater than AC-DC converter [20].

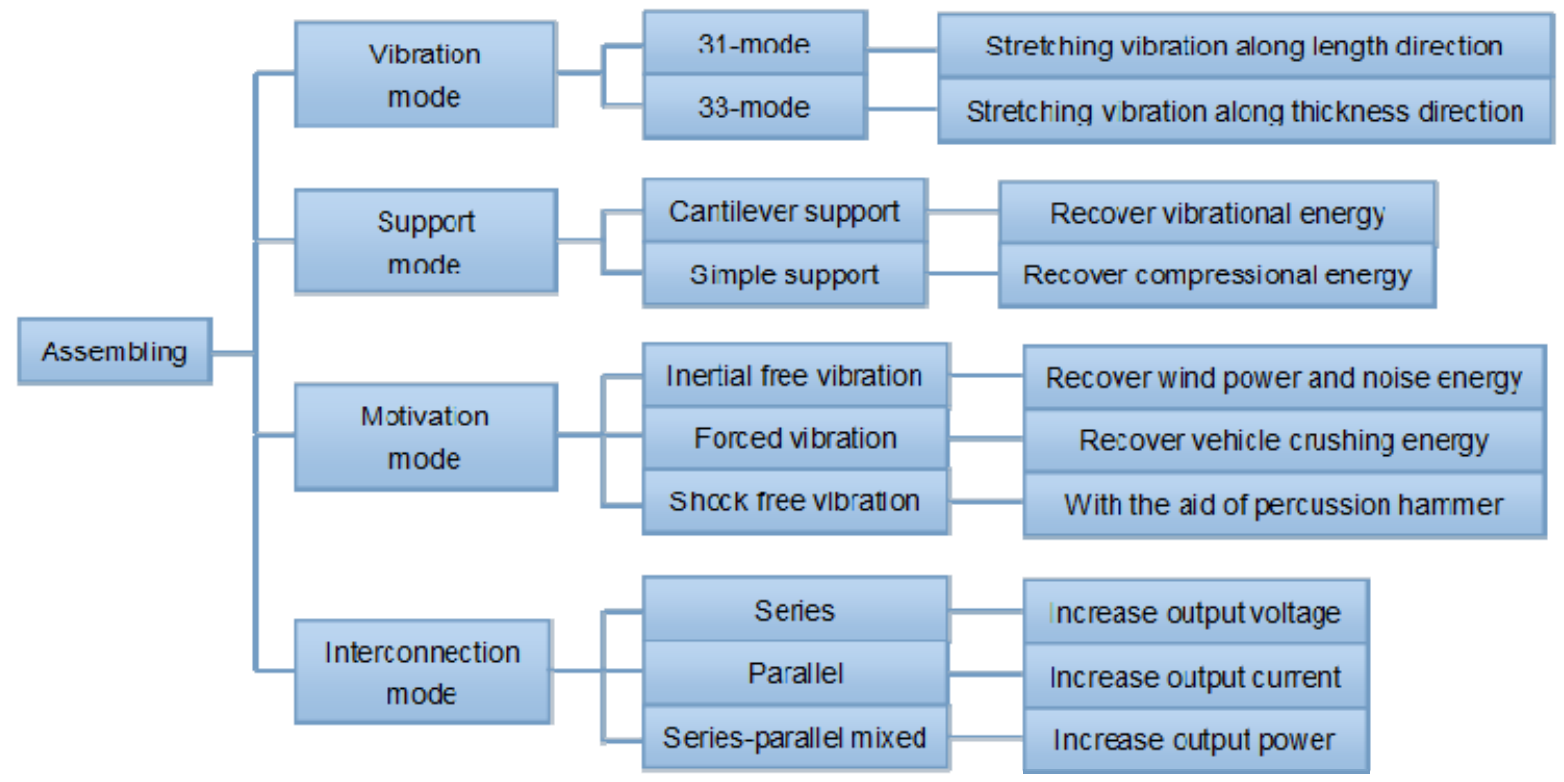

FIGURE I. ASSEMBLING ITEMS OF PIEZOELECTRIC POWER GENERATION EQUIPMENT AND THE FUNCTIONS OF EACH ITEM 


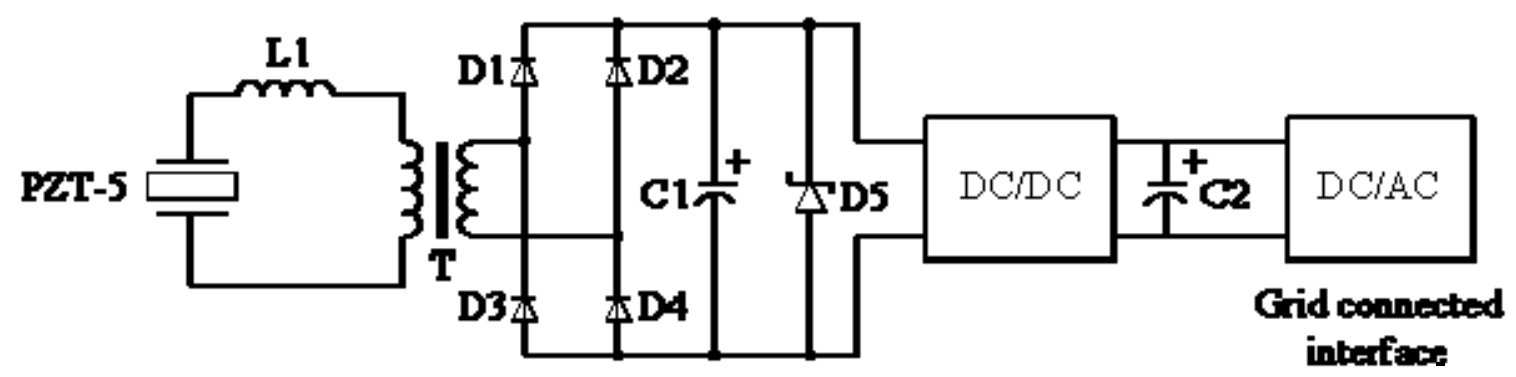

FIGURE II. OPERATING PRINCIPLE OF PIEZOELECTRIC POWER GENERATION EQUIPMENT

Electrolytic capacitor, supercapacitor, memory-free rechargeable battery [21] and many other devices can all serve as energy storage equipment. It is necessary to comprehensively assess these energy storage devices from experiments, determining their advantages and disadvantages.

Based on the foregoing discussions, the operating principle of piezoelectric power generation equipment is illustrated in Figure 2. When an intermittent external force is exerted on piezoelectric ceramic PZT-5, an alternating current with small current and high voltage is generated. To effectively collect the alternating current, an inductor L1 is combined with PZT-5 to form an LC resonance circuit. Because of the high voltage of the alternating current, it is lowered by a high frequency transformer $\mathrm{T}$ to a level that can be processed safely by the succeeding circuits. Next, the alternating current is rectified to a pulsating direct current by the rectifier bridge consisting of 4 fast recovery diodes D1 D4 and stored in the electrolytic capacitor $\mathrm{C} 1$. The zener diode D5 is used to further ensure the safety of the system. Considering the pulsatility of the direct current on $\mathrm{C} 1$, it is further processed by a $\mathrm{DC} / \mathrm{DC}$ circuit to a more stable one and then stored in the energy-storage capacitor $\mathrm{C} 2$. Finally, the energy stored in $\mathrm{C} 2$ can be transmitted to the power grid through a DC/AC interface.

\section{CONCLUSIONS}

The current researches related to piezoelectric power generation mainly concentrate in the fields of material science, mechanical science and microelectronic science, and certain limited and special application areas, e.g. self-powered wireless sensor network, piezoelectric road signs, piezoelectric power generation shoes, passive safety-belt detecting device and piezoelectric micro-electro-mechanical (MEM) system. This paper actually presents a prospect of the possible application of piezoelectric power generation in electricity production field. Except the important topics discussed in this paper, as the subsequent researches start, there will generate many other interesting problems worthy of researching.

\section{ACKNOWLEDGMENT}

This research is supported by Yongchuan District Science and Technology Committee Natural Science Fund Project (Ycstc, 2016nc3001)

\section{REFERENCES}

[1] International Energy Agency (IEA), in World Energy Outlook 2014, London, Nov. 2014.
[2] Y. Qiao, Z.X. Lu and F. Xu, et al, "Performance Evaluation Method of Wind-Coal Coordinating Operation, in Automation of Electric Power Systems," Vol. 37, No. 17, Sept. 10, 2013, pp. 26-33.

[3] Y.M. Park, N.H. Lee, T.R. Choung, "Effect of Noise and Low Frequency Noise Generated by Wind Power Plant (Wind Farm), in the 39th International Congress on Noise Control Engineering," INTERNOISE 2010, Lisbon, Portugal, 2010, Vol. 3, pp. 2061-2067.

[4] H. Zhang, X.R. Song and J. Feng, "Road Power Generation System Based on Piezoelectric Effect", Applied Mechanics and Materials, 2013, Vol. 329, pp. 229-233.

[5] Y. Jia and A.A. Seshia, "White Noise Responsiveness of an AIN Piezoelectric MEMS Cantilever Vibration Energy Harvester," Journal of Physics, 2014, Vol. 557, No. 1, pp. 25-33.

[6] T. Ashutosh and U. Lokman, Advanced Functional Materials, Wiley Scrivener Publishing, 2014

[7] K. Tomoyuki, Progress in Advanced Structural and Functional Materials Design, Springer Verlag, 2012.

[8] C. Lu, C.Y. Tsui and W.H. Ki, "Vibration Energy Scavenging System with Maximum Power Tracking for Micropower Applications," IEEE Transactions on Very Large Scale Integration (VLSI) Systems, Nov. 2013, Vol. 19, No. 11, 2109-2119.

[9] P. Li, F. Jin and J.S. Yang, "Effects of Semiconduction on Electromechanical Energy Conversion in Piezoelectrics," Smart Materials and Structures, Feb. 2015, Vol. 24, No. 2, pp. 210-218.

[10] O. Doaré and S. Michelin, "Piezoelectric Coupling in Energy- harvesting Fluttering Flexible Plates: Linear Stability Analysis and Conversion Efficiency," Journal of Fluids and Structures, Nov. 2011, Vol. 27, No. 8, pp. 1357-1375.

[11] Y. S. Li, W. J. Feng and Z. Y. Cai, "Bending and Free Vibration of Functionally Graded Piezoelectric Beam based on Modified Strain Gradient Theory," Composite Structures, Aug. 2014, Vol. 115, No. 1, pp. 39-47.

[12] S. Mohammadi and M.J. Abdalbeigi, "Analytical Optimization of Piezoelectric Circular Diaphragm Generator," Advances in Materials Science and Engineering, 2014, Vol. 16, pp. 46-60.

[13] C. Eichhorn, N. Tchagsim and N. Wilhelm, et al, "An Energyautonomous Self-tunable Piezoelectric Vibration Energy Harvesting System," Proceedings of the IEEE International Conference on Micro Electro Mechanical Systems, MEMS 2010, Cancun, Mexico, Jan. 2011, Vol. 1, pp. 1293-1296.

[14] V. R. Challa, M. G. Prasad and F. T. Fisher, "Towards an Autonomous Self-tuning Vibration Energy Harvesting Device for Wireless Sensor Network Applications," Smart Materials and Structures, Feb. 2011, Vol. 20, No. 2, pp. 81-91.

[15] C. G. Gregg, P. Pillatsch and P. K. Wright, "Passively Self-tuning Piezoelectric Energy Harvesting System," the 14th International Conference on Micro- and Nano- Technology for Power Generation and Energy Conversion Applications, PowerMEMS 2014, Awaji Island, Hyogo, Japan, Nov. 2014, Vol. 557, No. 1, pp. 52-59.

[16] J. J. Wang, Z. F. Shi and Z. J. Han, "Analytical Solution of Piezoelectric Composite Stack Transducers," Journal of Intelligent Material Systems and Structures, Sep. 2013, Vol. 24, No. 13, pp. 1626-1636.

[17] H. Q. Ye and R. M. Pan, "Experimental Analysis of Electro- mechanical Characterization of Piezoelectric Stack Actuators," Advanced Materials Research, 2011, pp. 293-297. 
[18] Y. P. Wu, A. Badel and F. Formosa, et al, "Piezoelectric Vibration Energy Harvesting by Optimized Synchronous Electric Charge Extraction," Journal of Intelligent Material Systems and Structures, Aug. 2013, Vol. 24, No. 12, pp. 1445-1458.

[19] Y. P. Wu, A. Badel and F. Formosa, et al, "Self-powered Optimized Synchronous Electric Charge Extraction Circuit for Piezoelectric Energy Harvesting," Journal of Intelligent Material Systems and Structures, Nov. 2014, Vol. 25, No. 17, pp. 2165-2176.

[20] Y. H. Su, Y. P. Liu and D. Vasic, et al, "Design of Piezoelectric Transformer-based DC/DC Converter to Improve Power by Using Heat Transfer Equipment," the 23rd International Conference on Adaptive Structures and Technologies, ICAST 2012, Nanjing, China, Oct. 2012, Vol. 1, pp. 90-95.

[21] Y. Levron, D. Shmilovitz and S.L. Martinez, "A Power Management Strategy for Minimization of Energy Storage Reservoirs in Wireless Systems with Energy Harvesting," IEEE Transactions on Circuits and Systems I: Regular Papers, 2011, Vol. 58, No. 3, pp. 633-643. 